\title{
Analysis of Ar Impurity Transport in the Large Tandem Mirror Device GAMMA 10/PDX Plasmas*)
}

\author{
Sayuri TAKECHI, Ryoko TATSUMI, Md. Shahinul ISLAM ${ }^{1)}$, Kazuo HOSHINO, \\ Akiyoshi HATAYAMA and Yousuke NAKASHIMA ${ }^{1)}$ \\ Graduate School of Science and Technology, Keio University, Yokohama 223-8522, Japan \\ 1) Plasma Research Center, University of Tsukuba, Tsukuba 305-8577, Japan
}

(Received 29 September 2018 / Accepted 25 December 2018)

\begin{abstract}
The purpose of this study is to understand the Ar impurity transport process in the GAMMA 10/PDX. In the present study, the initial simulation using LINDA code and IMPGYRO code has been done to establish the basis of further understanding of the Ar impurity transport. The validity of the simulation model has been discussed by comparisons with those by the theoretical estimations of the friction force and the thermal force. The simulation results show that the Ar impurities with $Z=1$ are transported towards the core region, since the thermal force dominates over the friction force under the present calculation conditions. The tendency does not contradict the experimental results and is reasonably explained by the theoretical prediction, i.e., the force balance between the thermal force and the friction force. The robust basis for further model validation and understanding of the $\mathrm{Ar}$ transport in the GAMMA 10/PDX has been established.
\end{abstract}

(C) 2019 The Japan Society of Plasma Science and Nuclear Fusion Research

Keywords: GAMMA 10/PDX, divertor, impurity transport, Ar gas, IMPGYRO

DOI: $10.1585 /$ pfr. 14.2403045

\section{Introduction}

Argon (Ar) impurity seeding to enhance the radiation energy loss in SOL/Divertor plasmas is one of the candidates to reduce the heat load on the divertor plates in the future fusion reactors such as DEMO [1]. However, if a large amount of the seeded impurities penetrates into the core plasma, the radiation cooling of the core plasma takes place and consequently, the nuclear fusion reactions stop. Therefore, it is important to understand and control the $\mathrm{Ar}$ impurity transport in the SOL/Divertor region.

The purpose of this study is to understand the Ar impurity transport in the linear experimental divertor simulator, the GAMMA10/PDX [2], by using the numerical simulation codes (the LINDA [3,4] code: Linear Divertor Analysis code and the IMPGYRO [5] code). Detailed comparisons of simulation results with those by the experiments seem to be relatively easy in such a simple linear geometry, and therefore it helps us understand the Ar transport phenomena in the SOL/divertor region of tokamaks.

The LINDA code is a two-dimensional (2-D) plasma fluid code for the linear divertor simulation devices like the GAMMA 10/PDX, while the IMPGYRO code is a kinetic impurity transport code based on the test particle model with Monte-Carlo methods for collisions. The IMPGYRO code has been originally developed for the high- $Z$ metal impurity ions like tungsten. In this study, the code has been extended so as to apply to the simulation of the $\mathrm{Ar}$

author'se-mail: takechi@ppl.appi.keio.ac.jp

*) This article is based on the presentation at the 12th International Conference on Open Magnetic Systems for Plasma Confinement (OS2018). impurities by implementing the Ar atomic data [6] into the code.

In the present calculations, the IMPGYRO code is applied to the GAMMA 10/PDX plasma under the trace impurity limit. In other words, the Ar impurity transport is calculated with the fixed background plasma profiles calculated by the LINDA code under the assumption that the Ar impurity densities are low enough compared with those of the background plasma.

The initial results are checked by comparisons with those by the simple theoretical formula to examine whether the results are both physically and numerically reasonable or not. These initial analysis and code validation are indispensable to establish the robust basis for more detailed comparisons with those by the experiments and to achieve the final goal, i.e., to understand the Ar transport process in the GAMMA 10/PDX.

\section{Simulation Model}

Detailed descriptions of the LINDA code and the IMPGYRO code have been given in Ref. [3, 4] and [5], respectively. Here, we briefly summarize the basic features of these codes. The LINDA code is a 2-D multi-fluid code, which has been developed based on the geometry of the GAMMA 10/PDX. The basic equations and the numerical method are almost the same as those of the B2 code [7]. The LINDA code outputs the plasma density $n_{\mathrm{e}}$, flow velocity parallel to the magnetic field lines $u_{/ /}$, electron temperature $T_{\mathrm{e}}$ and ion temperature $T_{\mathrm{i}}$. 


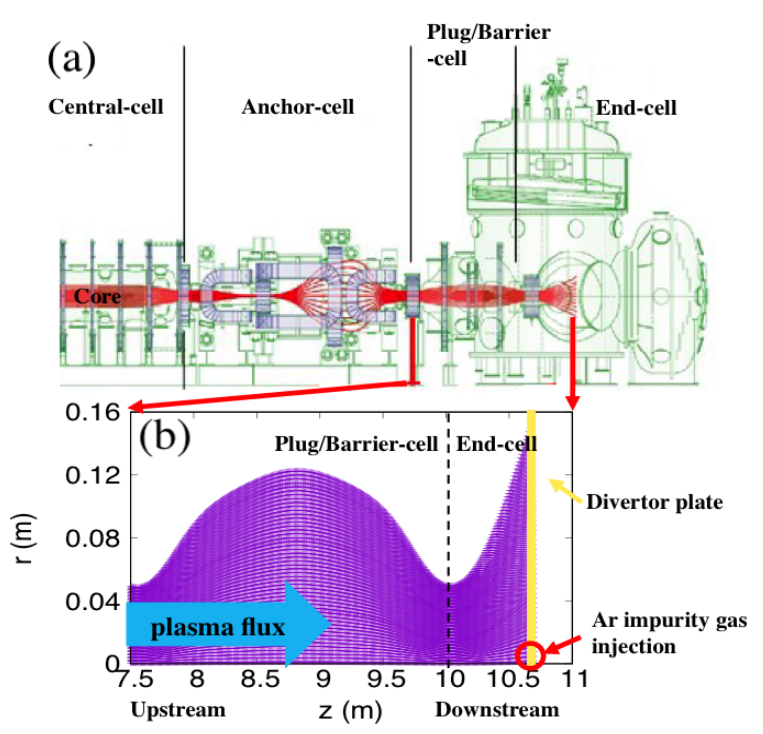

Fig. 1 (a) Schematic view of the GAMMA 10/PDX and (b) numerical mesh structure of the simulation region [4].

The IMPGYRO code is an impurity transport code which directly follows the gyro-motion of the Monte-Carlo test particles for each charge state without the guiding center approximation. The thermal force and the friction force due to Coulomb collisions are simulated by the Binary Collision Model (BCM) [8,9]. As for the Ar atomic process, (e.g., ionization and the recombination processes), two options exist to simulate them: 1) a conventional and standard simple "Hit or Miss" method, or 2) an "Implicit Monte Carlo" method [10]. In the present study, the first one, the Hit or Miss method, has been chosen.

Figures 1 (a) and (b) show the schematic view of the GAMMA 10/PDX and the numerical mesh structure of the simulation region, respectively. As seen from Fig. 1 (b), the simulation domain is set from the end-cell region to the plug/barrier-cell region. As the coordinate system, the cylindrical coordinate system $(r, \theta, z)$ has been used and the axial symmetry has been assumed $(\partial / \partial \theta=0)$. The origin $(z=0)$ of the $z$-axis is located at the center of the central cell region. The plug/barrier-cell and the end-cell correspond to the region from $z=7.5 \mathrm{~m}$ to $z=10 \mathrm{~m}$, and that from $z=10 \mathrm{~m}$ to $z=10.7 \mathrm{~m}$, respectively.

As shown in Fig. 1 (b), the numerical mesh structure of the simulation space has been generated based on the GAMMA 10/PDX magnetic configuration and each numerical mesh consists of the local orthogonal curvilinear system perpendicular and parallel to the magnetic field line. The totally $50 \times 320$ (50 in the radial direction and 320 in the axial direction) numerical grids have been used in the present simulation.

Figure 2 shows the 2-D profiles of typical plasma parameters obtained by the LINDA code. In this test calculation, relatively simple plasma profiles (see Fig. 2) have been given based on result of the LINDA code result in Ref. [3].
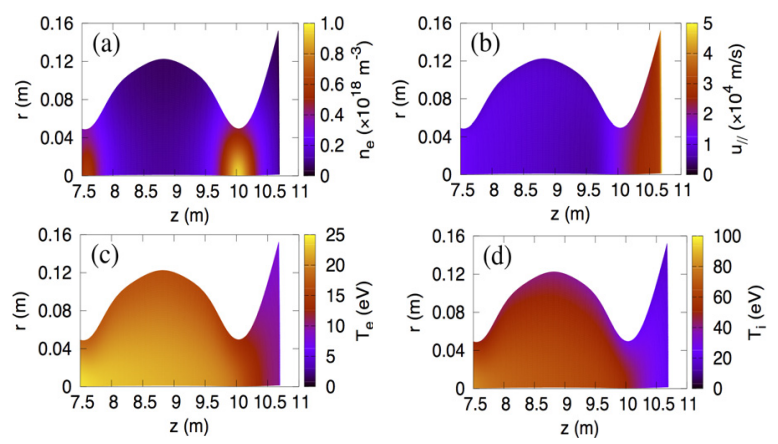

Fig. 2 2-D profiles of typical plasma parameters, (a) background plasma density $n_{\mathrm{e}}$, (b) background flow velocity $u_{/ /}$, (c) background electron temperature $T_{\mathrm{e}}$ and (d) background ion temperature $T_{\mathrm{i}}$.

Using these background profiles, we have done the impurity transport simulation with the IMPGYRO code. In the simulation the Ar test particles are injected from the location $(r, z)=(0.012 \mathrm{~m}, 10.68 \mathrm{~m})$ with a cosine angular distribution and a monotonic energy $(E=0.026 \mathrm{eV})$. The numerical time step for BCM is taken as $\Delta t=10^{-8} \mathrm{~s}$.

\section{Simulation Results and Discussion 3.1 Results of the Ar impurity transport}

Figures 3 and 4 show 2-D density profiles of the Ar impurity calculated under the background plasma profiles (given as Fig. 2). In Fig. 3 the Ar impurity density is summed up over all the charge states $Z(Z=0-18)$. On the other hand, Fig. 4 shows the Ar density profiles for each charge state, (a) $Z=0$, (b) $Z=1$, (c) $Z=2$ and (d) $Z=3$, respectively. For the better statistics, the density profiles in Figs. 3 and 4 have been averaged over the period of $100 \mathrm{~ms}$ after reaching the steady state. Besides, they are normalized by the maximum density in the calculation domain.

As seen from Figs. 3 and 4 (b), the Ar impurity ions are transported to the core region and this tendency is reasonably matches with experimental results $[11,12]$. The neutral Ar impurity particles do not exist in the plug/barrier-cell, because almost all the Ar particles are ionized, when passing through the position under the coil $(z \sim 10 \mathrm{~m})$ where the plasma density and temperature are high. The number of the Ar impurity $Z=1$ and 2 is large, while that of $Z=3$ is very small as shown in Fig. 4 .

\subsection{Analysis of Ar impurity transport}

Basically, the Ar impurity transport is determined by the thermal force and the friction force. It is difficult to separate the contribution from the thermal force $F_{\nabla k T}$ and the friction force $F_{0}$ in the IMPGYRO code which are automatically included in the Coulomb Collision model. Therefore, we use the analytic expressions [5] to estimate the contribution from each force, described by Eqs. (1) and (2), respectively. 


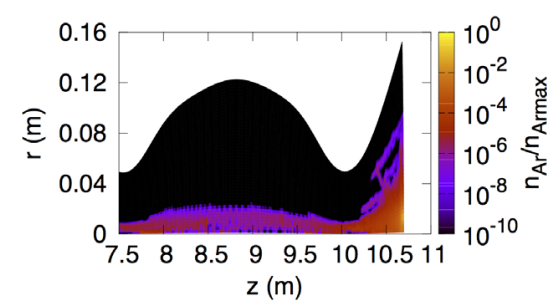

Fig. 3 2-D normalized density profile of Ar impurities. The total density of all the charge state $(Z=0-18)$ has been plotted by taking the time average of $100 \mathrm{~ms}$ in the steady state.
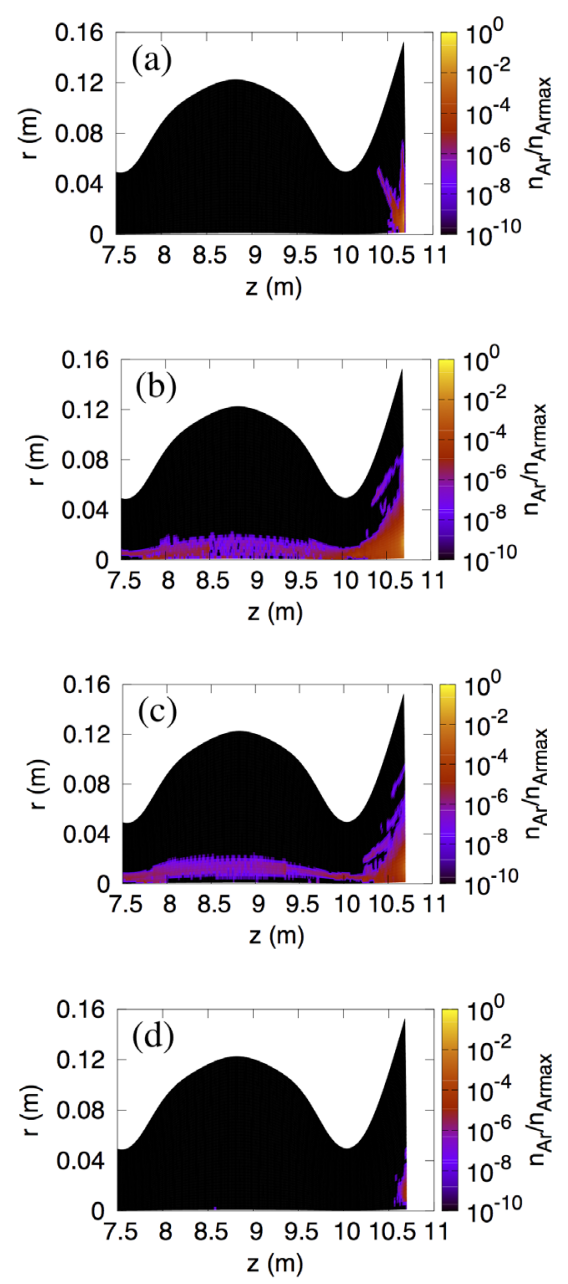

Fig. 4 2-D normalized density profiles of the Ar impurities for the charge state, (a) $Z=0$, (b) $Z=1$, (c) $Z=2$ and (d) $Z=3$, respectively. The densities are normalized and time is averaged in the same way as Fig. 3.

$$
\begin{aligned}
F_{\nabla k T}= & -\frac{C}{10 \pi \sqrt{\pi}} \sqrt{\frac{m_{\mathrm{i}}}{2 T_{\mathrm{i}}}} \frac{1}{T_{\mathrm{i}}^{2}} \exp \left(-\tilde{v}_{a}^{2}\right) \\
& \cdot\left[-\kappa_{/ /} \nabla_{/ /} T_{\mathrm{i}}-2\left(-\kappa_{/ /} \nabla_{/ /} T_{\mathrm{i}} \cdot \tilde{v}_{a}\right) \tilde{v}_{a}\right], \\
F_{0}=- & \frac{C}{4 \pi} \frac{n_{\mathrm{i}}}{2 T_{\mathrm{i}}} \frac{\Phi\left(\tilde{v}_{a}\right)-\tilde{v}_{a} \Phi^{\prime}\left(\tilde{v}_{a}\right)}{\tilde{v}_{a}^{3}} \cdot \tilde{v}_{a}, \\
\Phi(v)= & \frac{2}{\sqrt{\pi}} \int_{0}^{v} \exp \left(-x^{2}\right) \mathrm{d} x, \\
\Phi^{\prime}(v)= & \frac{\mathrm{d} \Phi(v)}{\mathrm{d} v}=\frac{2}{\sqrt{\pi}} \exp \left(-v^{2}\right),
\end{aligned}
$$

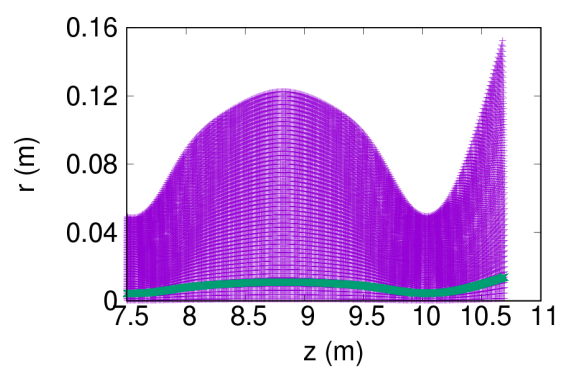

Fig. 5 Magnetic field lines in the calculation domain. The green thick line is the 5th mesh in the $r$ direction which is used for the analysis in Figs. 6 and 7.

where $C$ is a constant, $m_{\mathrm{i}}$ is the background plasma mass, $\tilde{v}_{a}$ is normalized relative velocity, $\kappa_{/ /}$is the heat conductivity, $\nabla_{/ /} T_{\mathrm{i}}$ is the background ion temperature gradient, and $n_{\mathrm{i}}$ is the background plasma density, respectively. From Eqs. (1) and (2), the thermal force is proportional to $\nabla_{/ /} T_{\mathrm{i}}$ and the friction force is proportional to $n_{\mathrm{i}}$.

In Fig. 5, the green thick line shows the magnetic field line passing near the point where the Ar impurity gas is injected in Fig. 1 (b). In the following, the Ar transport will be discussed with the green line. The horizontal axis is the length $L$ of the magnetic field line measured from the entrance of the plug/barrier region $(z=7.5 \mathrm{~m})$ in Fig. 1. Figure 6 shows (a) the thermal force and the friction force, (b) the total force (the thermal force + the friction force), and Fig. 7 shows average velocities of $\mathrm{Ar}$ ion (a) $Z=1$ and (b) $Z=2$, respectively. The signs of $F_{\nabla k T}$ and $F_{0}$ are defined according to the direction of the forces. If the force points to the divertor plate, then $F_{\nabla k T}, F_{0}>0$. If the force points to the core, then $F_{\nabla k T}, F_{0}<0$.

First, we focus on the transport process of the Ar impurity ions $(Z=1)$. As seen from Figs. 6 and 7 (a), we can summarize the results as follows:

1) Since the plasma density is low in the GAMMA 10/PDX, the thermal force strongly dominates over the friction force in the whole simulation space as shown in Fig. 6 (a).

2) As a result, the total force exerted on the Ar impurity ion becomes negative, i.e., towards the core region in most of the region (see Fig. 6(b)).

3) This total force accelerates the Ar ions towards the core region. As seen from Fig. 7 (a), the absolute value of the average velocity (which points to the core region) of the Ar impurities starts to increase around $L=2.7-2.8 \mathrm{~m}$. This can be explained by the total force in Fig. 6(b) whose absolute value increases around $L=2.7-2.8 \mathrm{~m}$ as well.

4) From these processes, 1), 2) and 3) above, the $\mathrm{Ar}$ impurity ions $(Z=1)$ are transported towards the core region.

On the other hand, the Ar impurity ions $(Z=2)$ have velocities toward the divertor plate $\left(v_{a}>0\right)$ at $L \sim 2.5 \mathrm{~m}$ as 

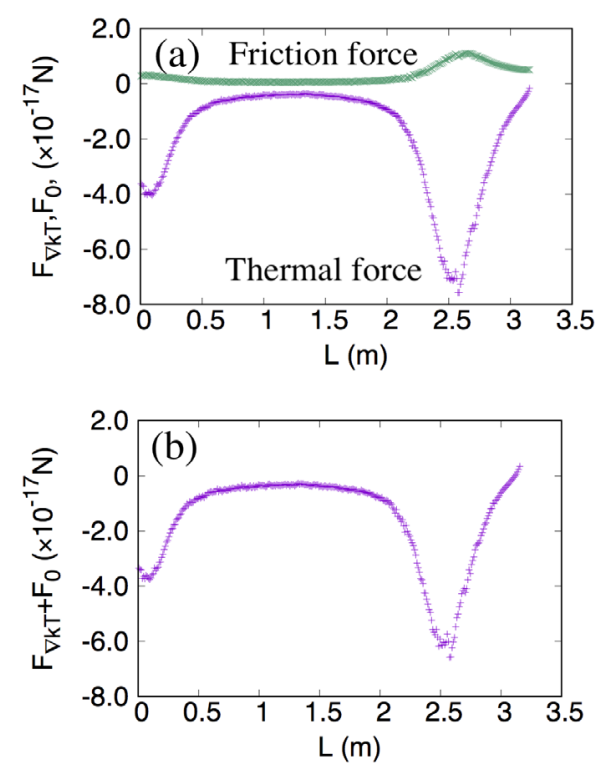

Fig. 6 Average forces acting on Ar impurity ions with $Z=1$ along the magnetic field line shown in Fig. 5 (green thick line): (a) the thermal force and the friction force, (b) the total force.
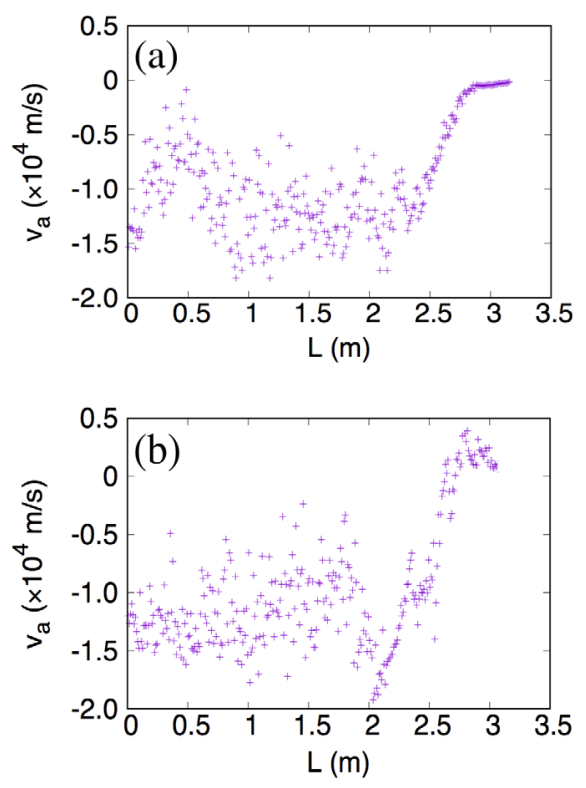

Fig. 7 Average velocities of Ar impurity ions with (a) $Z=1$ and (b) $Z=2$ along the magnetic field line shown in Fig. 5 (green thick line).

shown in Fig. 7 (b). This may be because the GAMMA $10 / \mathrm{PDX}$ has a mirror configuration. Therefore, it is possible that the Ar impurity ions with $Z=2$ are reflected around the end of the plug/barrier-cell at $L \sim 2.5 \mathrm{~m}(z \sim 10.0 \mathrm{~m})$ by the mirror force which are automatically included in
IMPGYRO code and they are ionized to $Z=3$ just in front of the divertor $L \sim 3.0 \mathrm{~m}(z \sim 10.5 \mathrm{~m})$.

It is important to consider the mirror force in the GAMMA 10/PDX different from tokamaks. Detailed analysis of the mirror force will be discussed in the future.

\section{Summary and Future Plan}

Ar impurity transport in the plug/barrier-cell and the end-cell region of the GAMMA 10/PDX has been calculated by means of the IMPGYRO code and the LINDA code in the trace impurity limit.

The initial results have been checked by comparisons with analytic expressions for the thermal force and the friction force. Regarding the Ar impurity ions with $Z=1$, the spatial profiles of Ar density and velocity are reasonably explained by the force balance between the thermal force and the friction force along the magnetic field line. Their tendencies do not contradict experiments $[11,12]$ as well.

From these initial results and their validity check, robust basis for further model improvements and detailed comparisons with experimental results have been established towards the final goal, i.e., understanding of $\mathrm{Ar}$ transport process in the GAMMA 10/PDX.

Towards the final goal, the following improvements and analyses will be necessary in the future. In order to compare the simulation results more directly and more in detail with those in the experiments, calculation of the spectral intensity of Ar I and Ar II in the plug/barrier-cell will be needed. In addition, the effects of the background density, mirror force and the divertor geometry on the Ar transport (V-shaped divertor plate) will be investigated in the future. Furthermore, integration of the IMPGYRO code into the LINDA code will be indispensable for more self-consistent simulations of the impurity transport in the background plasma without assuming the trace impurity limit.

[1] N. Asakura et al., Nucl. Fusion 53, 123013 (2013).

[2] Y. Nakashima et al., Nucl. Fusion 57, 116033 (2017).

[3] M.S. Islam et al., AIP Conf. Proc. 1771, 060015 (2016).

[4] M.S. Islam et al., Plasma Phys. Control. Fusion 59, 125010 (2017).

[5] S. Yamoto et al., Nucl. Fusion 57, 116051 (2017).

[6] H.P. Summers, Atomic data and analysis structure, Technical report, JET Joint Undertaking, Culham (1994).

[7] B.J. Braams, NET Rep. 68 (1987) EURFU/X -80/87/68.

[8] T. Takizuka and H. Abe, J. Comp. Phys. 25, 205 (1977).

[9] Y. Homma and A. Hatayama, J. Comp. Phys. 250, 206 (2013).

[10] A. Suzuki et al., J. Comp. Phys. 131, 194 (1997).

[11] T. Yokodo et al., Plasma Fusion Res. 13, 3402032 (2018).

[12] K. Shimizu et al., Fusion Sci. Technol. 68, 130 (2015). 